\section{Tail amputation in Indian Spectacled Cobra Naja naja - A case study}

\author{
S.S. Jadhav ${ }^{1}$, D.S. Disale ${ }^{1}$ and S. Mukherjee ${ }^{2}$ \\ ${ }^{1}$ Veterinarian, ${ }^{2}$ Wildlife Incharge, WILD WORLD, Animal HELP Foundation, \\ Ahmedabad, Gujarat, India \\ Email: ${ }^{2}$ alphamale84@ rediffmail.com
}

plus web supplement of 1 page

Indian Spectacled Cobra Naja naja is a smooth-scaled, black or dark brown to yellowish-white, medium sized snake, measuring about $1.5-2 \mathrm{~m}$. The underside is usually white or yellowish with a wide dark neckband. Hood marking and classic design shows a connected pair of rings. It is the most widely distributed species in India and is one of the 'big 4' important snakes. These cobras are common in rice growing areas that have plenty of rats for their food and holes to live in. Cobras are hunted and killed for their skin and out of fear. The Government of India has controlled export of Cobra skin and the species is now protected under Schedule II of the Indian Wildlife (Protection) Act (1972) (Whitaker, 2002). Around 700-800 snakes are rescued and rehabilitated by WILD WORLD of Animal HELP Foundation every year. Amongst these, 1$2 \%$ snakes have injuries over their body, incurred from people trying to kill them.

An adult male Indian Spectacled Cobra was rescued from 'ghatlodia' area of Ahmedabad city on 15 April 2007, with perceptible swelling over the tail region from the wrath of humans..

On clinical examination, prolapse of left hemipenis was observed (Image $1^{\mathrm{w}}$ ). Fracture of vertebral column behind the cloaca was discovered with a cripitation sound on palpation. No sensation was observed on the posterior fractured part of tail. So, based on clinical observation, it was decided to amputate the tail at the fracture site.

The head of Cobra was restrained by 'three finger grip' by a trained handler. Inj. meloxicam @0.2mg/kg body weight i/m (Bradey, 2001) was administered. Then anaesthesia was induced withinj. ketamine@ $30 \mathrm{mg} / \mathrm{kg}$ body weight $\mathrm{i} / \mathrm{m}$ (Bradey, 2001). After 10-15 minutes of anaesthetic injection, isoflurane was used to maintain the anaesthesia in boyl's apparatus.

Endotracheal tube No. 2.0 was passed through the larynx and anaesthesia was maintained with $3 \%$ isoflurane in 1.5 liters oxygen per minute. During the isoflurane anaesthesia the cobra was ventilated manually five times/minute by giving pressure on ventilation rubber bag. Monitoring of heart beat was done every five minutes to observe the condition of the snake. The surgical site was washed with normal saline solution and then sprayed with spirit (rubbing alchohol) and again painted with betadiene solution.

' $U$ ' shaped incision was made on the ventral and dorsal side of the tail at the fractured site. The skin was dissected bluntly to anterior part of surgical site (Image $2^{\mathrm{w}}$ ). The muscle was incised ventrally with help of surgical blade to open intravertebral space. This part was cut with a bone cutter. The right part of hemipenis was ligated with catgut no ' 0 ' and cut with a pair of scissors. The muscle was sutured with catgut no 'o' to close the bone part. Skin was sutured by horizontal mattress suture line with nylon.

The animal was dressed daily for seven days with betadine and acrillin ointment. Inj. enrofloxacin@ $@ 5 \mathrm{mg} / \mathrm{kg}$ body weight i/m (Divers, 1998) and inj. meloxicam @ $0.2 \mathrm{mg} / \mathrm{kg}$ body weight i/m were given for five days. After nine days after the wound had healed properly (Image $3^{\mathrm{w}}$ ) the snake was released back in wild.

${ }^{w}$ See Image $1-3^{w}$ in the websupplement at www.zoosprint.org

Manuscript 1789; (C) ZOO; Date of publication 21 November 2007 Received 25 May 2007; Finally accepted 30 September 2007
References

Whitaker R. (2002). Common Indian Snakes - A Field Guide. Macmillan India Ltd., pp.55-58.

Diver's S.J. (1998). Antimicrobials in reptiles. Exotic Veterinary Magazine (DVM) 1: 23.

Diver's S.J. (1999). Anaesthesia in reptiles. Exotic Veterinary Magazine (DVM) 1(3): 7-8.

Bradey Teresa (2001). Recognizing pain in exotic animals.

Exotic Veterinary Magazine (DVM) 3(3): 21-26.

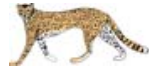

VET BRIEF

ZOOS' PRINT JOURNAL 22(12): 2950-2951

\section{Adynamic ileus in a tigress Panthera tigris}

\author{
I. Nath ${ }^{1}$, S.K. Panda ${ }^{2}$, N. Sahoo ${ }^{3}$, S.K. Rath ${ }^{4}$, P.K. Roy ${ }^{5}$ and \\ A.K. Mishra ${ }^{6}$
}

${ }^{1}$ Associate Professor, Department of Surgery, ${ }^{2}$ Assistant Professor, ${ }^{4}$ P.G Scholar, Department of Pathology, ${ }^{3}$ Associate Professor, Department of Medicine, Orissa Veterinary College, Bhubaneswar, Orissa 751003, India ${ }^{5}$ Senior Veterinary Officer, ${ }^{6}$ Assistant Director, Nandankanan Zoo, Baranga, Orissa, India

Email: ${ }^{1}$ indravet@yahoo.co.in

plus web supplement of 1 page

A Royal Bengal tigress "Devi” aged 17 years at Nandan Kanan zoo was noted to be off-feed for 48 hours. The animal had been kept in a squeeze cage for treatment of a chronic wound in the lumbar region (Image $1^{\mathrm{w}}$ ). The temperature and respiration rates were recorded to be $99.8^{\circ} \mathrm{F}$ and $14 / \mathrm{min}$. The case was referred to Orissa Veterinary College for treatment. On examination, the wound was found to be septic and extending about $6 \mathrm{~cm}$ into the muscles. The tigress was dull with posterior weakness. She was bearing weight in her hind limbs when excited. The sensory perception of hind limbs, perineum and tail was fair to painful stimulus. Sterile swabs were collected from the wound for culture and sensitivity test. Blood samples were also collected for haematological examination.

The wound was irrigated with $0.1 \%$ potassium permanganate lotion and dried with cotton swabs (Image $2^{\mathrm{w}}$ ). Povidone-iodine (Betadine lotion; Win-Medicare Pvt.Ltd., New Delhi) 5\% lotion was poured into the wound cavity. The tigress was administered with $500 \mathrm{ml}$ each of $5 \%$ Dextrose( D5), Ringers lactate (RLClaris Life Sciences Ltd., Ahmedabad), Rintose (Wockhard Ltd., Mumbai) through lateral coccygeal vein and two ampoules of Ranitidine $1 \mathrm{ml}$ inj. (Ranitin inj.; Torrent Pharma Ltd.,) two ampoules of Vitamin B-complex (Optineuron.; Lupin Lab.) 3ml inj. and $1 \mathrm{~g}$ of Cefotaxime sodium inj.(Taxim; Alkem Laboratories Ltd.,Mumbai) intramuscularly on 18.iv.2006 and continued for five days. After a week on 25.iv.2006 it was reported that there was improvement in the intake of meat to about $3 \mathrm{~kg} /$ day with healing of the wound, but there was absence of defecation since 18.iv.2006. Adynamic ileus was suspected.

Antibiotics were changed on the basis of sensitivity test. The tigress was treated with DNS 5\%, D5, RL, Rintose $500 \mathrm{ml}$ each along with Amikacin $1 \mathrm{gm}$ inj. (Mikacin; Aristo Pharmaceuticals), Optineuron $3 \mathrm{ml} x$ 2 ampoules, Ranitin $1 \mathrm{ml} \times 2$ ampoules and irrigation of wound with ciprofloxacin. Blood examination revealed haemoglobin, RBC, WBC, neutrophil, lymphocyte, monocyte and eosinophil to be $11 \mathrm{~g} \%, 6.5$ millions $/ \mathrm{mm}^{3}$, 13 thousands $/ \mathrm{mm}^{3}, 65 \%, 30 \%, 2 \%$ and $3 \%$, respectively with absence of any haemoparasite. Soap water enema was given of about 3l. The animal vomited with few nematodes in the vomitus. Hence, $3 \mathrm{ml}$ of Ivermectin inj. (Virbac Animal Health India Ltd.) was administered

w See Images in the websupplement at www.zoosprint.org

Manuscript 1712a; (C) ZOO; Date of publication 21 November 2007 Received 29 January 2007; Revised received 05 October 2007; Finally accepted 10 November 2007 